EGU2020-7382

https://doi.org/10.5194/egusphere-egu2020-7382

EGU General Assembly 2020

(c) Author(s) 2020. This work is distributed under

the Creative Commons Attribution 4.0 License.

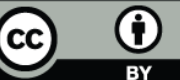

\title{
The oxidizing power of the dark side: Rapid nocturnal aging of biomass burning as an overlooked source of oxidized organic aerosol
}

John Kodros ${ }^{1}$, Dimitris Papanastasiou ${ }^{2}$, Marco Paglione ${ }^{3}$, Mauro Masiol $^{1}$, Stefania Squizzato ${ }^{1}$, Kalliopi Florou ${ }^{1}$, Agata Kołodziejczyk ${ }^{4}$, Ksakousti Skyllakou ${ }^{1}$, Athanasios Nenes ${ }^{1,5}$, and Spyros

Pandis 1,6

${ }^{1}$ Foundation for Research and Technology Hellas, Institute for Chemical Engineering Sciences, Patras, Greece (jkodros@iceht.forth.gr)

${ }^{2}$ Buffalo Research Laboratory, Honeywell International Inc.

${ }^{3}$ National Research Council, Institute of Atmospheric Sciences and Climate (CNR-ISAC), Bologna, Italy

${ }^{4}$ Institute of Physical Chemistry, Polish Academy of Science, Warsaw, Poland

${ }^{5}$ Laboratory of Atmospheric Processes and their Impacts, School of Architecture, Civil \& Environmental Engineering, École

Polytechnique Federale de Lausanne, Lausanne, 1015, Switzerland

${ }^{6}$ Department of Chemical Engineering, University of Patras, Patra, Greece

Oxidized organic aerosol (OOA) is a major component of ambient particulate matter, substantially affecting both climate and human health. A considerable body of evidence has established that $\mathrm{OOA}$ is readily produced in the presence of daylight, thus leading to the association of high concentrations of OOA in the summer or mid-afternoon. However, this current mechanistic understanding fails to explain elevated OOA concentrations during night or wintertime periods of low photochemical activity, thus leading atmospheric models to under predict OOA concentrations by a factor of 3-5. Here we show that fresh emissions from biomass burning rapidly forms OOA in the laboratory over a few hours and without any sunlight. The resulting OOA chemical composition is consistent with the observed OOA in field studies in major urban areas. To estimate the contribution of nocturnally aged OOA in the ambient atmosphere, we incorporate this nighttime-aging mechanism into a chemical-transport model and find that over much of the United States greater than $75 \%$ of the OOA formed from fresh biomass burning emissions underwent nighttime aging processes. Thus, the conceptual framework that OOA is predominantly formed in the presence of daylight fails to account for a substantial and rapid oxidation process occurring in the dark.

How to cite: Kodros, J., Papanastasiou, D., Paglione, M., Masiol, M., Squizzato, S., Florou, K., Kołodziejczyk, A., Skyllakou, K., Nenes, A., and Pandis, S.: The oxidizing power of the dark side: Rapid nocturnal aging of biomass burning as an overlooked source of oxidized organic aerosol, EGU General Assembly 2020, Online, 4-8 May 2020, EGU2020-7382,

https://doi.org/10.5194/egusphere-egu2020-7382, 2020 
